\title{
HOMOGENEIDAD DEL LÉXICO AGRÍCOLA EN LA MERINDAD DE CAMPOO SEGÚN EL ATLAS LINGÜÚISTICO Y ETNOGRÁFICO DE CANTABRIA
}

\author{
JOSÉ MANUEL RUIZ NÚÑEZ \\ (Universidad de Alicante)
}

\begin{abstract}
RESUMEN
In spite of its small dimensions, Cantabria has suffered a remarkable process of comarcalización (regionalization), reflected on its linguistic and cultural diversity. In this article we seek to emphasize the homogeneity of the agricultural vocabulary of Merindad de Campoo, a southern Cantabrian district, in contrast to the rest of the region; in addition to this, it is noticed the increasing incursion of the Castilian variety into the dialectal one. So as to prove both issues, we have analysed the materials of the Allas linguiustico y etnografico de Cantabria (The Linguistic and Ethnographic Atlas of Cantabria) by Manuel Alvar from a comprehensive point of vue, but paying special attention to the aspects that link Linguistic Geography with Lexicography.
\end{abstract}

\section{Introducción}

Nuestro trabajo se encuadra dentro de la tercera etapa de la geografía lingüistica' y se centra en el análisis del léxico agrícola de la Merindad de Campoo $^{2}$ según el Atlas lingüístico y etnográfico de Cantabria ${ }^{3}$, cuyo lenguaje

Las tres ctapas a las que nos referimos son: $I^{a}$ ) recolección del material mediante encuestas basadas en un cuestionario, $2^{\mathrm{a}}$ ) registro de dichos materiales en los mapas, esto es, la confección de los atlas lingüísticos y $3^{a}$ ) estudio e interpretación de los datos allegados (Coseriu 1977: 113-1 14).

2 Esta comarca corresponde a las siguientes localidades del Atlas lingüístico y efnográfico de Cantabria (ALECant) (vid. mapa 2): S 313, 409, 500, 501, 502, 503, 504, 600 у 601, respectivamente: Abiada, La Población del Yuso, Fresno del Río, Villanueva, Olea, Aldea de Ebro, San Andrés de Valdclomar, Polientes y Villaescusa de Ebro.

3 Sobre el cuestionario, la realización y las característisticas del ALECant, vid. Alvar (1979: 81-118; 1991: 349-402; 1994: 41-79; 1995: 7-33), Alvar y Nuño (1981: 359-370), Alvar y García Mouton (1985: 194-197), García Mouton (1987:65; 1994: 157-158) y Gimeno (1990: 89). 
popular ${ }^{4}$ «es una joya inestimable» en opinión de Julio Montes Sáiz (Calderón Escalada 1981: vii). Esta comarca ${ }^{5}$, antiguamente ocupada por los cántabros (García de Diego 1946: 191), está situada en el alto Ebro y ocupa el sur de la región; queda separada de la mitad norte por la Cordillera Cantábrica y de La Liébana, al oeste, por la Sierra del Cordel; su poblamiento es concentrado (González Echegaray y Díaz Gómez 1988: 20-21, 61). Precisamente, los mapas del ALECant ponen de manifiesto la existencia de dos áreas contrapuestas: la septentrional frente a la meridional (Alvar 1991: 371-372). Por último, en el mapa dialectal de García Lomas (1966) la zona 5 coincide con esa comarca (vid. mapa 1), donde predomina la influencia del castellano antiguo. No es extraño, pues, que la Merindad de Campoo constituya una zona individualizada, compacta, y que ello se relleje en la homogeneidad de su vocabulario. Sin embargo, no se trata de una comarca aislada, sino que mantiene vínculos en cuanto a la lengua, las costumbres y el folclore con Liébana y Pernía, con las tierras de Aguilar de Campoo y con algunos valles de la cabecera de Burgos; en efecto, muchas de las voces que se emplean en la comarca sureña se atestiguan en el resto de la comunidad cántabra, en Palencia e incluso en León y Burgos (Calderón Escalada 1981:2).

Pese a la notoria variedad lingüística de Cantabria (Alvar 1991: 371-372), en Campoo existe una notable homogeneidad en cuanto al vocabulario agrícola. Así

4 Calderón Escalada (1981: 2-24) estudia las peculiaridades linguísticas de la Merindad de Campoo. En cuanto al lconés, donde se suele incluir el dialecto de la Montaña, vid. García de Diego (1946: 135-194), Menéndez Pidal (1962: 13-117), Zamora Vicente (1979: 84-210), Lapesa (1988: 477-492) y Borrego Nieto (1996: 139-158). Sobre la aspiración de la $h$ y la metafonía vocálica cn el norte peninsular: Menéndez Pidal (1954), Rodríguez Castellano $(1954,1959)$ y Alonso (1962). Sobre el neutro de materia: Gareía González (1989). Acerca del dialecto de la Montaña: Menéndez Pidal (1973:93-94), García de Dicgo (1946: 191-194), García Lomas (1966: 21 -53), González Echegaray y Díaz Gómez (1988: 30, 48-54), Renero Díaz (1947: 111-216), Múgica (1892: 1-40), Alvar (1995: 814, 26-33) y Nuño Álvarez (1996: 183-196). Sobre el habla pasiega: García Lomas (1966: 87-93; 1977: 71 -75) y Penny (1969); sobre Tudanca: Penny (1978). Los dos estudios de Penny dedican sendos capítulos a la metafonía en Cantabria.

Si bien la extensión territorial de Cantabria es muy reducida $\left(5.289 \mathrm{~km}^{2}\right)$, su realidad física ha favorccido cnormemente el proceso de comarcalización cultural que la caracteriza. Se trata de una zona montañosa, donde abundan las pequeñas aldeas más o menos aisladas y los núcleos urbanos son de poca importancia; sólo las localidades costeras contaron con una verdadera comunicación natural. De ahí, que Alvar (1995: 13) relacione este hecho con la riqueza del vocabulario de la antigua provincia de Santander. No obstante, Cantabria ha conservado su unidad en tanto que región historica: por una parte, ha existido siempre como país, desde antiguo llamado Cantabria, cuyos habitantes han tenido conciencia de formar una entidad propia; por otra, en la actualidad ocupa un área mís reducida que en el pasado, pero ésta constituye el núcleo sustancial de la región a lo largo de su historia. Además, como ha defendido Caro Baroja (1977), los actuales pueblos del norte peninsular, a los cuales pertenecen los cántabros, son culturalmente descendientes de los antiguos: ni la romanización, ni las inmigraciones, ni los trasiegos de gentes a consecuencia de la Reconquista han anulado la peculiaridad cultural de aquéllos (González Echegaray y Díaz Gónez 1988: 19, 33 34, 61). Por último, Tovar (1955:9, 33) afirma que la lengua de los indoeuropeos contribuyó en buena medida a la configuración histórica de este pueblo. 
pues, en estas páginas vamos a analizar aquellas denominaciones de uso generalizado en la mencionada comarca que, además, la particularizan frente al resto de la región

A la hora de comentar los materiales seleccionados del ALECant adoptaremos un punto de vista amplio: aparte de referirnos a la distribución geográfica de las voces, darcmos cabida a aquellos aspectos que vinculan la geografía lingüística con otras disciplinas afines como son la etimología, la etnografía, la semántica y, en especial, la lexicografía.

\section{Análisis de mapas referidos a la agricultura}

2.1. Con el significado de 'erial' (ALECant I, 125) predomina bald(r)ío ${ }^{7}$, que es la voz característica de Campoo. Segû́n la compilación académica, baldio es 'la tierra que ni se labra ni está adehesada', definición a la que remite Calderón Escalada (1946, 1981). Asimismo, se registra en el ALEA (I, 7) (Fernández Sevilla 1975: 19-21), en puntos sueltos del ALEANR (I, 11 ) (Garcés Gómez 1984: 20-28), en Las Palmas y en Hierro (ALEICan I, 8) ${ }^{8}$; en Miguélez Rodríguez ${ }^{9}$ consta la variante baldiyu.

2.2. Para designar la "puerta hecha a manera de verja que cierra los huertos, corrales o jardines' (DRAE, s. v. cancilla) se utiliza comúnmente la voz portillera $^{10}$ (ALECant I, 141). Según el Diccionario Académico, portillera es 'paso de entrada en las fincas rústicas'. López Vaqué recoge esta voz como 'portilla grande de una casa o corralada'. En el habla pasiega purtillera equivale a 'cancilla con varales sueltos que se quitan y se ponen' (Penny 1969: $\$ 261$ ). GDiego (1985)" la registra en tanto que 'puerta rústica'. En asturiano se emplean portellera/-u y portilleru con el valor de 'cancilla'. Se trata, pues, de un

6 En el ALECant, existen 55 puntos de encuesta. De todos ellos, 9 corresponden a Campoo. Por tanto, consideramos que un término es característico de esta comarca cuando consta en cuatro o más localidades y no ligura, o lo hace sólo esporádicamente, en el resto de Cantabria.

En Campoo baldio en S 409 (baldrío), 500, 501, 502, 503, 504; en el resto de Cantabria en S 307. De aquí en adelante indicamos entre paréntesis las variantes de la voz que se cita como entrada o como variante más frecuente, además de incluir determinados detalles que consignan los encuestadores para algunas palabras. Igualmente, señalamos en nota las localidades cántabras no pertenecientes a la comarca de Campoo en que se registra el término en cuestión.

8 Citamos los otros atlas como ALEA, ALEANR y ALEJCan, respectivamente: Alvar (con la colaboración de A. Llorente y G. Salvador) (1961-1973), Alvar (1975-1978) y Alvar (con la colaboración de A. Llorente, T. Buesa y E. Alvar) (1979-1983).

9 Los vocabularios y recopilaciones de términos dialectales se citan abreviadamente por el nombre del autor cuando éste tiene un único trabajo.

10 En Campoo portillera en S 500, 503, 504, 600 (portilleras), 601; en el resto de Cantabria en S 408. Campoo no es una zona yeísta (Calderón Escalada 1981: 7-8), pero este fenómeno, corriente en la región cántabra, ya se detecla en la comarca que estudiamos (cfr. portiya en S 501, en este mismo mapa y sayar en $\mathrm{S} 502$ en $\$ 2.5)$.

11 G-Diego = García de Diego (1985). 
deslizamiento semántico. En el ALEANR $(I, 26)$ se respondió portillera en Rioja (Garcés Gómez 1984: 274).

2.3. 'Levantar los rastrojos con el arado' se denomina levantar ${ }^{12}$ y arromper $^{13}$ (ALECant I, 144). El Diccionario Académico no da ninguna acepción específica para levantar, sino la general 'recoger o quitar una cosa de donde está. Díez Suárez (1994: 52) registra levantar (s. v. alza) en Argüellos como 'la primera reja o vuelta que se da al rastrojo' y en el Luna como 'la última reja'; Miguélez Rodríguez recoge levantar como 'realizar la primera operación a la tierra para prepararla para la siembra'. Se ha producido, pues, una restricción de significado. Arromper es un término familiar con el significado de 'romper o roturar' (DRAE) o un arcaísmo con a protética (Renero Díaz 1947: 205); en asturiano arromper y romper equivalen a 'romper, roturar la tierra'. En el ALEA (I, 18) hay 69 localidades con la respuesta levantar, pero no está generalizada en toda la región sino que predomina en el este; en 18 pueblos se registra romper, casi todos de Granada y de Almería (Fernández Sevilla 1975: 42-50). En el ALEANR (I, 28) romper es general en Navarra y Rioja, y se halla en gran parte de Huesca y en puntos aislados de Zaragoza y Teruel; levantar se localiza en Navarra y Rioja (Garcés Gómez 1984: 93-95, 282). En el ALEICan (I, 21) romper (y variantes) se registra en poblaciones aisladas del archipiélago, excepto en Hierro, y levantar sólo en localidades aisladas de Tenerife y Gomera.

2.4. La 'mata de espinos sobre la cual se colocan unas piedras para que hagan peso y que se arrastra para igualar el terreno o extender el estiércol' se llama basnial $^{14}$ (ALECant I, *149). Esta voz está suficientemente atestiguada por los vocabularios dedicados al dialecto de la Montaña, aunque no siempre con epéntesis de $i$. En el léxico oficial encontramos basna como 'especie de narria, cajón o escalera de carro, para llevar arrastrando cosas de gran peso' y en Tudanca como 'narria que sirve para acarrear hierba, tirada por bueyes. Tiene forma triangular y consiste en la horca de un árbol con varias tablas superpuestas, todas sujetadas al ápice de la horca' (Penny 1978: § 283). López Vaqué (s. v. basna) añade a la definición del DRAE que la basna es una "especie de narria o rastra $\sin$ ruedas que se emplea para extender las toperas o el abono en los campos y para bajar la hierba de los sitios altos'. Según González Echegaray y Díaz Gómez (1988: 106), basna es 'narria en que se cargan las hierbas tras la siega'. Saiz Barrio registra basna con dos significados: 'rastra sin rueda a modo de trineo para transportar principalmente hierba por los lugares más abruptos' y 'conjunto de ramas o espinos atados por un extremo, al que se coloca algún peso

12 En Campoo levantar en S 313, 409, 500, 502; en el resto de Cantabria en S 304. En S 601 icncmos levantar la ristrojera como perífrasis de 'barbechar', donde rastrojera vale por 'conjunto de tierras que han quedado de rastrojo' (DRAE).

1.3 En Campoo arromper en S 501, 503, 600, 601; en el resto de Cantabria en S 311 (romper).

If En Campoo basnia en S 313,501, 502, 503, 504; en el resto de Cantabria en S 306, 308, 311. 
encima (piedras generalmente) y que enganchado a una yunta se utiliza para desmenuzar el abono previamente esparcido en el prado'. Calderón Escalada (1946, 1981) define basnia como 'basna. Narria. Instrumento de labranza que, arrastrado sobre los prados y las tierras, sirve para igualar su superficie y repartir el estiércol y esparcir las toperas. Rastra de ramas para arrastrar pedruscos, etc.'. Saiz Barrio incluye basnia (junto a basna) como 'rastra confeccionada con espinos o ramas que, arrastrada sobre un prado o tierra, sirve para igualar su superlicie, desmenuzar el estiércol, esparcir toperas, etc. Igualmente se usa para cl transporte de alguna piedra u otro peso'.

2.5. 'Escardar a mano' es sallar' ${ }^{15}$ en la comarca de Campoo (ALECant I, 153). En el habla pasiega sallar y en Tudanca sayar se usan con la acepción de 'escardar por primera vez' (Penny 1969: $§ 270$; 1978: § 306). Según la compilación académica, sallar ${ }^{16}$ es 'cavar con azada o azadilla'. G-Diego localiza esta voz en Asturias, Burgos y Cantabria; COR $^{17}$ añade Vizcaya y parte de Asturias; Neira y Piñeiro citan sallar, sayar, satsar y sachar como 'escardar' en asturiano. Vergara Martín (1921: 96) recoge sallar como 'volver a escardar los trigos para quitar las hierbas que nacen en los sembrados. En algunos puntos, cavar alrededor de la caña del maíz'. García Lomas recoge sall/yar como sinónimo de 'sachar, escardar'. López Vaqué define sallar (s. v. sallo) como 'sachar; escardar la tierra sembrada para quitar las malas hierbas, a fin de que prosperen las plantas útiles', acepción que coincide con la de Saiz Barrio. Por último, Bartolomé Suárez (1993: 255) dice de sallar 'labor del campo que consiste en arrimar tierra a la raíz de las plantas hortenses para fortalecerlas. 'También sayar'.

2.6. El verbo que se utiliza con el significado de 'escardar con herramienta' es escavar' (ALECant I, 154). En el DRAE consta dicha voz como 'cavar ligeramente la tierra para ahuecarla y quitar la maleza'. Según Calderón Escalada (1981), en Campoo escavar vale por 'escardar, entresacar las malas hierbas, cavando', tal y como muestra el mapa. En leonés escarvar tiene la acepción de 'aricar la tierra, escavanar' (Miguélez Rodríguez). Si comparamos este mapa con cl precedente ( $\$ 2.5$ ), se observa que siempre existe un lexema diferente para cada uno de los sememas en la comarca del sur cántabro: sallar 'escardar a mano' frente a escavar 'escardar con herramienta'.

2.7. Para referirse a la 'época en que se ara' se emplean con frecuencia estas dos denominaciones: primavera y adviento (ALECant I, 148). La voz

15 En Campoo sallar en S 500, 502 (sayar), 503, 504, 600, 601; en el resto de Cantabria en S 203 (styar), 308, 309 .

16. En el Diccionario de Autoridades la voz sallar ya aparecía con la marca diatópica referida a la Montaña (Salvador Rosa 1985: 117).

17 COR $=$ Corominas y Pascual (1980-1991).

18 En Campoo escavar en S 500, 502, 503, 504, 600, 601; en el resto de Cantabria en S 102, 407. 
predominante y exclusiva de Campoo es primavera ${ }^{19}$; en Miguélez Rodríguez posec el significado de 'primer pasto de los prados de regadío'. En el sur de esta comarea se silúa adviento ${ }^{20}$. Se trata de meras referencias temporales que no aparecen en la compilación oficial con la acepción que aquí estudiamos.

2.8. El 'azadón de peto' se llama normalmente azada de peto ${ }^{21}$ (ALECant I, 128), voz que no consta en el Diccionario de la Academia. En el habla pasiega se registra azá de petu 'azadón de peto' (Penny 1969: § 240). García Lomas (s. v. peto) afirma que el azadón de peto "se llama así por semejanza del peto o cabo que liene la podadera, por la parte opuesta, en figura de formón".

2.9. La "herramienta cuyas dos brocas tienen forma de pico' recibe la denominación pica ${ }^{22}$ (ALECant I, *192). Pica se halla, además, en localidades aragonesas y tinerfeñas (crr. ALEANR I, *104; ALEICan I, *19). El DRAE no registra pica como 'pico' sino como 'escoda ${ }^{23}$ con puntas piramidales en los cortes, que usan los canteros para labrar piedra no muy dura'. Para García Lomas signilica 'pico de afilar el dalle' y 'punterola de cantero'. En el habla pasiega, pica es 'alcotana' ${ }^{24}$ (Penny 1969: $\$ 368$ ) y en Campoo 'martillo de afilar el dalle' (Calderón Escalada 1981). En asturiano pica vale por 'herramienta de labrador con dos puntas opuestas, una generalmente plana, la otra afilada' (Neira y Piñeiro). Creemos que en pica 'herramienta cuyas dos brocas tienen forma de pico' hay un simple cambio de género, rasgo típico del dialecto montañés (García Lomas 1966: 26).

2.10. El 'arado de madera' se denomina arao romano ${ }^{25}$ (ALECant I, 178); como 'arado de cama' consta en Cantabria (González Echegaray y Díaz Gómez 1988: 126) ${ }^{26}$. La especificación romano, que acompaña a arao, remite a la procedencia de tal apero. En el $A L E A(\mathrm{I}, 136)$, donde se preguntó simplemente

1) En $S 409,500$ (se siembra en octubre), 501, 502, 601

20) En S 502 (arada adelantada en Navidad), 503 ("octubre o noviembre"), 504 (arviento, como 502), 601; G-Diego localiza la variante arviento en la Rioja.

21 En Campoo azada de peto en $S 409,500,501,502,503,600$; en cl resto de Cantabria en S 100,209 (azada con peto), 404 (id.).

22 En Campoo pica en 501, 502, 503, 504, 600,601; en el resto de Cantabria en S 306.

23 Escoda es 'herramienta en forma de martillo, con corte en ambos lados, para labrar piedras y picar paredes' (DRAL).

24 Según el DRAE, alcotana significa therramienta de albañilería, que termina por uno de sus extremos en figura de azuela y por el otro en figura de hacha, y que tiene en medio un anillo en que entra y se asegura un mango de madera, como de medio metro de largo. Hay algunas con boca de piqueta, en ve\% de corte'.

25 En Campoo arao romano en S 313 (aráu romanu), 409, 500, 501, 502, 503, 504, 600, 601; en el resto de Cantabria en S 304, 307 (aráu romano), 308 (aráu romanu), 310 (íd.). Aráu 'arado' es la Forma corriente en asturiano (Neira y Piñeiro).

20. La partc fundamental del arado romano (tipo 3), el más corriente en Castilla y conocido con tal nombre, es la cama. Los arados del valle de Campoo son arados-cama (tipo 3) de «timón compucsto» de dos piezas; cabe destacar la forma en que está colocada la mancera y el volumen del dental, que, junto al timón compuesto, se hallan también en Palencia y en el norte de Burgos (Caro Baroja 1983: 515, 531). 
por 'arado', cncontramos arado romano en varios puntos de Almería y Córdoba, y como respuesta aislada en Huelva, Granada y Jaén (Fernández Sevilla 1975: 416-419). En el ALEANR (I, 132) dicha voz se registra un par de veces en Logroño, además de aparecer en sendas poblaciones de Soria y Cuenca.

2.11. El 'palo derecho que sale de la cama del arado y al que se fija el tiro' (DRAE, s. v. timón) se llama (ar)rastradero ${ }^{27}$ (ALECant I, 181). En el Diccionario Académico encontramos arrastradero, mas no con el significado que nos interesa. García Lomas recoge arrastradera/-o como 'pieza del aladro al extremo del timón' y rastradera, variante con aféresis de $a$-, como 'arrastradera o delantera del timón del arado'. Calderón Escalada (1946, 1981) define arrastradero (con - u final en Campoo) como 'pieza del arado romano que va al extremo donde se coloca el yugo de la yunta', tal como hace Saiz Barrio. Dicho término adquicre $\mathrm{cl}$ sentido de 'timón' porque tal palo 'arrastra' las otras piczas que componen el arado. Puesto que (ar)rastradero no se transcribe en los otros atlas que manejamos, podemos afirmar que se trata de una voz exclusiva de la comarca de Campoo.

2.12. La 'clavija del timón' se denomina llavija ${ }^{28}$ (ALECant I, 183). Esta variante palatalizada de la voz castellana clavija no figura en el DRAE. Como obscrvamos, en Campoo, y en general en el dialecto de la Montaña, triunfa la evolución CL-> $/ l$ incluso en los casos en que nuestra lengua se muestra conservadora". La voz llavija consta en García Lomas y Saiz Barrio como "pieza pequeña de madera que hace las veces de llave y de pina; clavija', en Huidobro y en Calderón Escalada $(1953,1981)$ como mero sinónimo de clavija, en Renero Díaz como 'clavija, especialmente la llave de madera que cierra la cebilla de los ganados', cn el habla pasiega como 'mano de la torga, que sirve para cerrarla' (Penny 1969: \$285) y en Bartolomé Suárez (1993: 239) como 'uno de los componentes de la cabilla o prisión'. Yavija aparece en ocho localidades de Jaén (ALEA I, *140) (Fernández Sevilla 1975: 421) y frecuentemente en Logroño (ALEANR I, 138); la variante llavía 'clavija' se documenta en bable (Neira y Piñciro).

2.13. El 'conjunto de filamentos de la espiga' (DRAE, s. v. argaya) es la garabasta ${ }^{3 / 1}$ (ALECant I, *155). El léxico oficial localiza garabasta en Cantabria como 'argaña de trigo', esto es, como sinónimo de la voz general del español argayalargaña. Garabasta figura en Calderón Escalada (1953) como 'cada una de las aristas de la espiga del cereal', en Saiz Barrio como 'arista o cascarilla de los cereales' y en G-Diego como 'granzas de cereales' en Burgos y Cantabria. De

27 S 409, 500, 501, 502 (rastradero), 503, 504 (id.), 600-601 (restradero).

2s En Campoo en S 500, 501, 502 (llavijo), 503, 504, 600,601 (id.); en el resto de Cantabria yavija en S 100, 101, 104.

2) Esto fenómeno se documenta sobradamente en Cantabria y, en concreto, en Campoo. Cfr. García de Diego (1946: 157), Renero Díaz (1947: 121), García Lomas (1966: 40) y Calderón Escalada (1981:7).

3) S $501,502,503,504,600,601$. 
acuerdo con el ALECant, garabasta se restringe a la comarca de Campoo y así lo confirman García Lomas, Calderón Escalada (1981) y López Vaqué. En cuanto a la etimología, Alvar (1991: 61, nota 98) afirma que las propuestas de G-Diego ( $\angle$ GRAMEN) y de COR (cfr. agavanzo) son poco convincentes. Nuevamente, estamos ante un término peculiar de la zona estudiada, pues no se registra en los demás atlas consultados.

2.14. Para referirse a 'cada una de las fundas de cuero o de otra materia, que se ponen [los segadores] en los dedos para que no se lastimen o manchen' (DRAE, s. v. dedil) encontramos la voz zoqueta ${ }^{31}$ (ALECant I, *156). Esta denominación fïgura como respuesta aislada en Jaén y Almería (ALEA I, 38), y es voz generalizada en el $A L E A N R(I, 54)$ como 'zoqueta del segador' pero no con el significado de 'dedil del segador' (ALEANR I, *54). Zoqueta consta en la compilación académica como término restringido a Aragón, Navarra y Rioja con la acepción de 'especie de guante de madera con que el segador resguarda de los cortes de la hoz los dedos meñique, anular y corazón de la mano izquierda'; COR (s. v. zoquete) no ofrece más información al respecto. El Diccionario Académico debería corregir esa marca diatópica, pues zoqueta también se registra en Campoo; asimismo, figura en Calderón Escalada (1981) y Saiz Barrio como 'estuche de madera para resguardar el filo o corte de las herramientas' y más especílicamente como 'pequeño estuche de madera que sirve para proteger los dedos de la mano izquierda mientras se siega' en González Echegaray y Díaz Gómez. (1988: 127). Estamos, pues, ante un mero sustituto de los dediles de segador.

2.15. El 'mangote del segador' se denomina manguito ${ }^{32}$ (ALECant $\mathrm{I}$, *156). Según el $D R A E$, manguito vale por 'manga sobrepuesta para preservar la ropa', sin relerirse específicamente al que utiliza el segador, de ahí que se produzca un simple deslizamiento semántico (Alvar 1991: 75). En el ALEA (I, 39) figura manguito en bastantes puntos de Jaén y como respuesta aislada en Cádiz y Córdoba. En el ALEANR (I, 55), es voz de uso corriente en Aragón y Navarra, mientras que sólo consta en dos localidades logroñesas, en tres pueblos de la provincia de Castellón, dos de Valencia, dos de Cuenca y uno de Vitoria.

2.16. Para referirse al 'puñado de mies' predomina la voz mana(da) $)^{33}$ (ALECant I, *156). El léxico oficial recoge manada con el significado que nos interesa: "porción de hierba, trigo, lino, etc. que se puede coger de una vez con la mano'. En Lamano Beneite, manada es 'el puñado de espigas segadas, que deja en el surco el segador, y que va después la atariña recogiendo en gavillas para formar el haz'. En García Lomas encontramos maná con una acepción derivada de la del español general: 'se decía de cuatro señoritas o haces que se ataban con

\footnotetext{
$S 501,502,600,601$.

En Campoo en S 500, 501, 503, 504, 600, 601; en el resto de Cantabria en S 309.

33 En Campoo manada en S 502,503,504, 600,601; en el resto de Cantabria en S 311 (manió).
} 
el lino mismo, y que se podían coger con una mano'. En Andalucía, mana(da) se usa cn el 20\% de la región (Fernández Sevilla 1975: 148): es muy abundante en Jaén, Granada y Almería, pero se emplea de modo restringido en Sevilla, Córdoba y Huelva, y aisladamente en Málaga (ALEA I, 41). En el ALEANR (I, 57) se transcribe en pocos puntos de Navarra, Huesca y Zaragoza, además de una localidad conquense. En las Islas Canarias mana(da), general en Gomera, se halla también en Hierro, Las Palmas y Tenerife.

2.17. Cuando la parva está trillada y recogida se dice que está hecha ${ }^{34}$ (ALECant, lám. 86). Parva hecha consta en el ALEA (I, 63) (Fernández Sevilla 1975: 206). En este caso encontramos un verbo de significado muy amplio que adquicre un valor mucho más concreto.

2.18. Existen dos términos con el significado de 'semilla de la almorta': tito $^{35}$ y muela (ALECant I, *202). Según el DRAE, ambas voces son simples sinónimos de almorta; las dos se emplean en Tudanca (Penny 1978: $\$ 311$ ); tito ${ }^{36}$, además, sc localiza cn Sahagún y Vega de Esla (Díez Suárez 1994: 80) y muela en Castilla la Vicja (COR, s. v. almorta). En el ALEA ( $1, * 110)$ se transcribe muela como voz característica de la Andalucía occidental (Fernández Sevilla 1975: 239). En el ALEANR (I, 112), muela predomina en Logroño, pero también se registra en dos puntos de Navarra y en uno de Vitoria. De acuerdo con los materiales de los atlas españoles, tito $^{37}$ no podría considerarse voz de la lengua común como se desprende del léxico olicial, pues no hay rastro de ella ni en Andalucía, ni en el ALEANR, ni tampoco en la mayor parte de Cantabria.

2.19. El 'Salvado basto' se llama tercerilla ${ }^{38}$ (ALECant I, *251). Se trata de un nombre comercial, según anotan los encuestadores del atlas. La voz posee en el léxico oficial la accpción de 'salvado, cáscara del trigo', no exactamente 'salvado basto', por lo que se ha producido una restricción de significado. Por otra parte, tercerilla consta como 'harina de muchas mezclas' (Saiz Barrio) y 'salvado fino mezclado en parte con harina' (Miguélez Rodríguez). Terc/serilla 'salvado basto' sólo se halla en dos puntos de Huesca (ALEANR II, 238).

2.20. La 'cascarilla de los cereales' se denomina vaso ${ }^{39}$ (ALECant I, *155). En el Diccionario de la Academia vaso vale por 'pieza cóncava de mayor o menor tamaño, capaz de contener alguna cosa'. Estamos, pues, ante una metáfora evidente: gracias al sema 'contenedor', vaso pasa a significar 'cascarilla, corteza de los cereales'. En las hablas leonesas vaso consta exactamente como 'cápsula

$34 \mathrm{~S} 313,409,500,501,502,503,504,601$. En todos estos puntos coexiste con la voz general trillada.

$35 \mathrm{~S} 501,502,504,600,601$; fito coexiste con muela en las tres últimas localidades.

36 Tïo 'grano o chocho de las legumbres', 'guisante', 'pepita de cereza, pera o manzana' (Miguélez Rodríguez).

COR (s. v. tití) afirma, sin más, que tito significa 'almorta' en muchas partes de nuestro territorio linguístico.

38 En Campoo en $S 409,502,503,504,600,601$; en el resto de Cantabria en S 405, 407.

$39 \mathrm{~S} 501,504,600,601$. 
que envuelve el grano de los cereales' (Miguélez Rodríguez). En el ALEA (I, 36) se recoge vaso 'cascarilla del trigo' aisladamente en Huelva y Sevilla (3 puntos), pero la variante con diminutivo vas/cillo, típicamente occidental, aparece abundantemente en Cádiz y en Málaga, sobre todo en el oeste de esta provincia, además de figurar en Sevilla, en el norte de Córdoba y en un pueblo de Huelva (Fernández Sevilla 1975: 221). En varias localidades de Logroño se encuentra vaso como 'cascarilla del trigo', aparte de puntos sueltos de Navarra, Burgos y Vitoria.

2.21. La 'cáscara tierna y larga en que están encerradas las semillas de algunas plantas' (DRAE, s. v. vaina) se llama jeruga (ALECant I, 203). Ni jeruga ni jaruga están incluidas en la compilación académica; tampoco oruga consta en el $D R A E$ con ese significado, sino como 'planta herbácea anual, de la familia de las crucíferas, con tallos vellosos de cuatro a cinco decímetros de altura, hojas lanceoladas y partidas en varios gajos puntiagudos, flores axilares y terminales de pétalos blancos con venas moradas, y fruto en vainilla cilíndrica, con semillas globosas, amarillentas y menudas'. Calderón Escalada $(1953,1981)$ dice de jaluga o haluga 'vaina de la leguminosa'; lo mismo afirma García Lomas, quien localiza en Liébana todas estas variantes jaluga (también en Campoo), saluga, aluga, seluga y siluga; Saiz Barrio (s. v. aluga) añade otra: siruga; la variante s/ceruga aparece en bastantes puntos de Logroño y en un pueblo navarro, en tanto que oruga solamente se transcribe en una localidad burgalesa (ALEANR I, 115). Por último, Díez Suárez (1994: 69) registra jaluga 'vaina del haba' en Sajambre. Los tres primeros autores proponen el étimo SILICUA, -AE, que conserva la $s$ en lugar de la $j$ como rasgo típico del dialecto de la Montaña (Múgica 1892: 16; Renero Díaz 1947: 129-130; García Lomas 1966: 42). En lo que se refiere a la etimología, tanto G-Diego (s. v. orujo) como COR (s. v. oruga) proponen el término VÔLÛC(Û)LUM (de VOLVERE) 'envoltorio', que daría el antiguo y dialectal borujo 'orujo'. Fernández Sevilla (1975: 239-243) ha encontrado jaruga y jeruga en 15 puntos dispersos de Jaén, Granada y Almería (cfr. ALEA I, 111); dicha localización geográfica lo lleva a pensar en un posible arabismo jarruba 'algarroba' que, por extensión, vendría a significar 'vaina'. A nuestro juicio existe otra posibilidad más viable: además de transcribirse en Campoo las variantes jaruga, jeruga y oruga (ésta también en un pueblo de Burgos), se atestiguan formas con $s$ - inicial en varios léxicos cántabros y en el ALEANR; por consiguiente, creemos que podría tratarse de un cruce entre oruga ( $\angle$ ERUCA), cuya semilla también se halla dentro de una vaina pequeña, y SILIQUIA "vaina de cualquier fruto' (la $s$ - inicial se mantendría o evolucionaría a $j$-como es habitual en castellano).

4i) S 313 (oruga), 500-502 (jaruga), 503-601 (jeruga). Cfr. con aluga (y var.) en S 108 (scilugal), 308, 309 . 
2.22. La 'vaina seca' se denomina jeruga secal (ALECant I, 204). Por tanto, no existe un lexema específico para cada uno de estos sememas: la 'vaina de las legumbres' ( $\$ 2.21)$ se diferencia de la 'vaina seca' gracias a un adjetivo que funciona como especificador.

2.23. Las 'vilortas, abrazaderas del arado' (DRAE, s. v. belorta) reciben el nombre arquillos ${ }^{42}$ (y variante arcos) (ALECant I, *181). Arquillo significa 'abrazadera de metal o cuero que se engancha en el tranco para cerrar la puerta' (Miguélez Rodríguez). Esta voz no aparece en la compilación oficial, pero resulta cvidente que como diminutivo de arco 'aro que ciñe y mantiene unidas las duelas de pipas, cubas, etc.' (DRAE) o arcu 'arete metálico de refuerzo del zueco que tienc hendidura' en Tudanca (Penny 1978: § 375) pasa a significar fácilmente 'pieza de metal u otra materia que sirve para asegurar alguna cosa, ciñéndola' (DRAE, s. v. abrazadera). Arquillo sólo figura en una localidad de Gran Canaria y la variantc arquitos en otra de Hierro, pues en las Islas Canarias la denominación común es arco(s).

\section{Conclusiones}

El análisis realizado nos permite verificar fundamentalmente que la Merindad de Campoo, comarca sureña que corresponde a la zona 5 del mapa dialectal de García Lomas (1966) (vid. mapa 1), es compacta y particular en cuanto al léxico agrícola. En ella se utilizan las siguientes denominaciones: adviento/primavera 'nombre que dan a la época en que se ara' ( $\$ 2.7$ ), arao romano 'arado de madera' (\$1.10), arquillos 'vilortas' (\$2.23), (ar)rastradero 'timón' (\$2.11), arromperflevantar 'barbechar' (\$2.3), azada de peto 'azadón de peto' ( $\$ 2.8$ ), bald(r)ío 'crial' (\$2.1), basnia 'mata de espinos que se arrastra' $(\$ 2.4)$, escavar 'escardar con herramienta' ( $\$ 2.6)$, garabasta 'conjunto de filamentos de la cspiga' (\$2.13), hecha '(parva) recogida y trillada' ( $\$ 2.17)$, jeruga 'vaina de las lcgumbres' (\$2.21), jeruga seca 'vaina seca' (\$2.22), llavija 'clavija del timón’ $(\$ 2.12)$, mana $(d a)$ 'puñado de mies' $(\$ 2.16)$, manguito 'mangote del segador' ( $\$$ 2.15 ), muela/tito 'almorta' ( $\$ 2.18)$, pica 'pico' ( $\$ 2.9)$, portillera 'cancilla' ( $\$$ $2.2)$, sallar 'escardar a mano' (\$2.5), tercerilla 'salvado basto' (\$2.19), vaso 'cascarilla de los cereales' ( $\$ 2.20$ ) y zoqueta 'dedil del segador' ( $\$ 2.14)$.

Asimismo, hay loda una serie de voces agrícolas del español estándar que se utilizan normalmente en la comarca del sur cántabro, mientras que en el resto de la región bien se desconocen, bien se emplean muy aisladamente. Esto confirma que Campoo es una de las zonas más castellanizadas de Cantabria (vid. mapa 1).

41 En Campoo en S 313 (oruga), 500-502 (jaruga seca), 503-601 (jeruga seca); en el resto de Cantabria en S 307 (jaruga). Cfr. con aluga (y vars.) en S 107, 108 (saluga), 308-309 (aluga seca).

42 En Campoo arquillos en 500 (arcos), 502, 503, 504, 600; en el resto de Cantabria en S 311 , $312(\operatorname{arcos} s)$. 
A continuación enumeramos dichos términos: ahechar ${ }^{4.3}$ 'íd.' (ALECant I, 166), colmar ${ }^{44}$ 'íd.' (ALECant I, 169), colmo ${ }^{45}$ 'colmo de una medida' (ALECant I, 168), delantal ${ }^{46}$ 'mandil del segador' (ALECant I, *156), granza ${ }^{47}$ 'granzas' (ALECant I, 167), mediana ${ }^{48}$ 'id.' (ALECant I, 173), mies(es) $)^{49}$ 'trigal' (ALECant I, lám. 86), parva (50 'íd' (ALECant I, *164), tamo' 'íd' (ALECant I, lám. 86), tresnal ${ }^{52}$ 'íd.' (ALECant I, lám. 86) y veza ${ }^{53}$ 'íd.' (ALECant $\left.\mathrm{I}, * 202\right)$.

En fín, el propósito fundamental de este artículo no ha sido otro que poner de manifiesto la unidad y peculiaridad del léxico de la agricultura en la Merindad de Campoo, en el que se percibe claramente el proceso avanzado de castellanización, apoyándonos en los materiales del ALECant, materiales muy valiosos de los que ya no se puede prescindir ${ }^{54}$.

\section{MAPA DIALECTAL DE CANTABRIA (GARCÍA LOMAS 1996)}

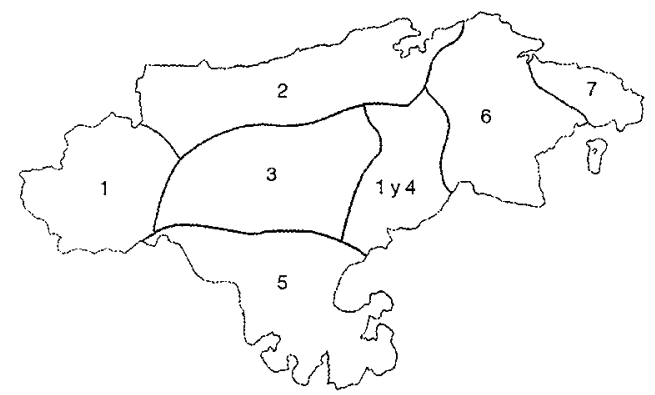

1.- Habla lebaniega con reminiscencias del leonés. 2.- Substratos del bable no absorbidos por la romanización. 3.- Influencia del latín vulgar, presencia de arcaísmos y fonética típica del dialecto montañés. 4.- Habla pasiega cuya fonética se emparenta con el bable y el leonés. 5.- Predominio del castellano antiguo en su invasión desde el sur hacia el norte. 6.- Confluencia de matices dialectales sin características peculiares. 7.- Tenues sedimentos translaticios del vasco.

4.3 En Campoo ahechar en S 409, 500, 501-502 (ahechear) 503, 504; en el resto de Cantabria en $\mathrm{S} 107$.

if $S 409,500,501,504,600,601$.

4.5 En Campoo colmo en S 409, 500, 501, 600 (colme); en el resto de Cantabria en S 402.

46. $\mathrm{S} 501,502,503,504,600,601$.

47 En Campoo en $S 409,501,502$; en el resto de Cantabria en S 303.

$48 \quad S 313,503,504,600,601$.

4) $\mathrm{S} 503,504,600,601$.

50 En Campoo en $S 313,409,500,501,502,503,504,600,601$; en el resto de Cantabria en $S$ 303.

51 En Campoo en $S 313,409,500,501,502,503,504,600,601$; en el resto de Cantabria en $S$ 312.

2 En Campoo en S 313, 409, 500, 501, 502, 503, 504, 601; en el resto de Cantabria en S 309.

5. En Campoo en S 500, 501, 502, 503, 504, 600, 601; en el resto de Cantabria en S 100.

5.4 Sobre la necesidad de analizar los atlas lingüísticos vid. Salvador (1985: 144), García Mouton (1990: 28), Alvar (1991: 114) y Navarro Carrasco (1993: 89-90, 93). 


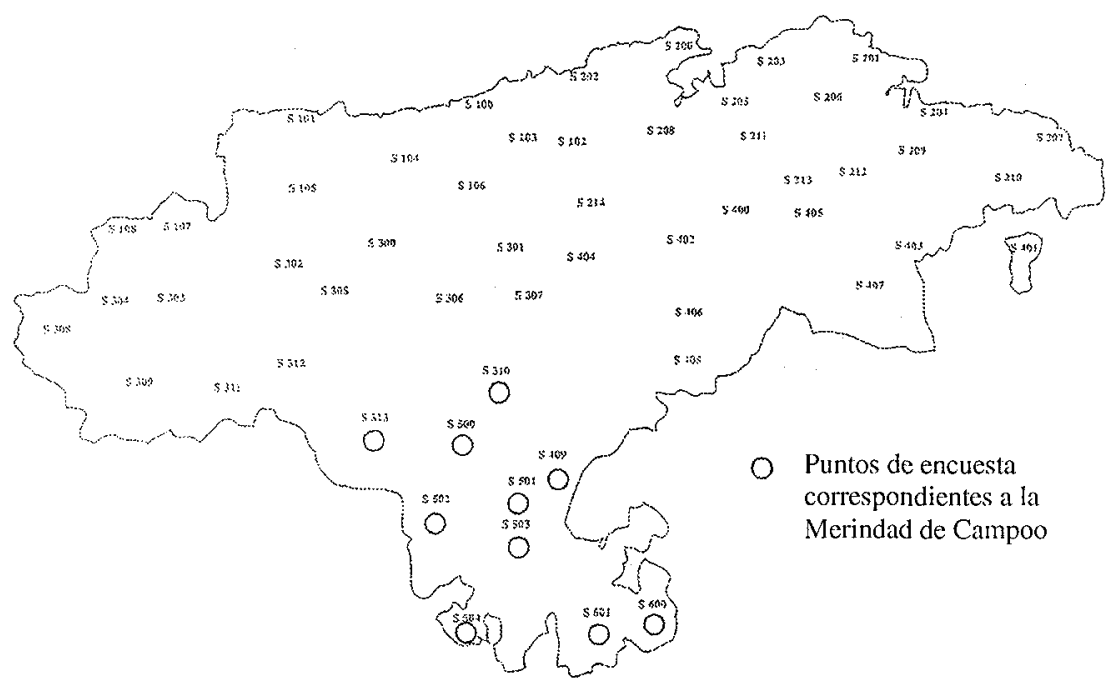

\section{Referencias bibliográficas}

ALONSO, DÁMASO (1962), «Metafonía, neutro de materia y colonización suditaliana en la península hispánica», Enciclopedia lingiiística hispánica, tomo I (Suplemento), Madrid: CSIC, págs. 105-154.

ALVAR, MANUEL (1975-1978), Atlas lingüístico y etnográfico de las Islas Canarias, 3 tomos, Las Palmas: Ediciones del Excmo. Cabildo Insular.

- (1979), «El Atlas lingüístico y etnográfico de la provincia de Santander (España)», RFE, LXI, págs. 81-118; posteriormente en Alvar, Manuel (1991), págs. 349-378 y como nota preliminar del primer tomo del ALECant, págs. 7-15.

- (1982), «Atlas linguísticos y diccionarios», LEA, IV, págs. 253-323; también en Alvar, Manuel (1991), págs. 49-115.

- (1991), Estudios de geografía lingüística, Madrid: Paraninfo.

- (1991), «Un ejemplo de atlas lingüístico automatizado: el ALES», en Alvar, Manuel (1991), págs. 379-402.

- (1994), «Atlas lingiiístico y etnográfico de Cantabria: Cuestionario» en García Mouton, Pilar (ed.) (1994): págs. 41-78.

- (dir.) (1996), Manual de dialectología hispánica. El Español de España, Barcelona: Ariel. 
- (con la colaboración de A. Llorente y G. Salvador) (1961-1973), Atlas Linguïístico y Etnográfico de Andalucía, $1^{\mathrm{a}}$ edición, Granada: UniversidadCSIC, 6 tomos.

- (con la colaboración de A. Llorente, T. Buesa y E. Alvar) (1979-1983), Atlas Lingiüistico y Etnográfico de Aragón, Navarra y Rioja, Zaragoza: Departamento de Geografía Lingüística, Instituto Fernando El Católico de la Excma. Diputación de Zaragoza, CSIC, 12 tomos.

- (con la colaboración de C. Alvar y J. A. Mayoral) (1995), Atlas lingiuístico y etnográfico de Cantabria, Madrid: Arco/Libros, 2 tomos.

- y García Mouton, Pilar (1985), «El Atlas lingüístico y etnográfico de Cantabria», Gran Enciclopedia de Cantabria, Santander: Editorial Cantabria, págs. 194-197.

- y Nuño, María Pilar (1981), «Un ejemplo de atlas lingüístico automatizado: el ALES», LEA, III, págs. 359-370.

BARTOLOMÉ SUÁREZ, ANTONIO (1993), Aforismos, giros y decires en el habla montañesa, Santander: Universidad de Cantabria.

BORREGO NIETO, JULIO (1996), «Leonés» en Alvar, Manuel (dir.) (1996): págs. 139-158.

CALDERÓN ESCALADA, JOSÉ (1946), «Voces, en su mayor parte nombre de cosas, de uso corriente en estos valles altos de la provincia de Santander, que no están recogidas en el Diccionario de la Lengua Española», BRAE, XXV, págs. 379-397.

- (1953), «Voces, en su mayor parte nombre de cosas, de uso corriente en estos valles altos de la provincia de Santander, que no están recogidas en el Diccionario de la Lengua Española», BRAE, XXXIII, págs. 295-304.

- (1981), Lenguaje popular de la Merindad de Campoo, Santander.

CARO BAROJA, JULIO (1977), Los pueblos del Norte, $3^{a}$ ed., San Sebastián: Ed. Txertoa.

- (1983), «Los arados españoles», Tecnología popular española, Madrid: Ediloria Nacional, 1983, págs. 509-597.

COROMINAS, JUAN Y J. A. PASCUAL (1980-1991), Diccionario crítico etimológico castellano e hispánico, 6 tomos, Madrid: Gredos.

COSERIU, EUGENIO (1977), El hombre y su lenguaje (Estudios de teoría y metodología lingiiistica), Madrid: Gredos.

DÍEZ SUÁREZ, Ma . SOLEDAD (1994), Léxico leonés, León: Universidad.

FERNÁNDEZ SEVILLA, JULIO (1975), Formas y estructuras en el léxico agrícola andaluz. Interpretación y estudio de 200 mapas linguiísticos, Madrid: CSIC.

GARCÍA GONZÁLEZ, FRANCISCO (1989), «El neutro de materia», en Abad, Francisco, Buesa, Tomás et alii, Homenaje a Alonso Zamora Vicente (Dialectología. Estudios del Romancero), Madrid: Castalia, 2 tomos: tomo 2, pp. 91-105. 
GARCÉS GÓMEZ, MARÍA PILAR (1984), Constitución histórica y estructura actual del léxico aragonés: agricultura y ganadería, Madrid: Universidad Complutense.

GARCÍA DE DIEGO, VICENTE (1946), Manual de dialectología española, Madrid: Espasa-Calpe.

- (1985), Diccionario etimológico español e hispánico, $2^{\mathrm{a}}$ ed., Madrid: Espasa-Calpe.

GARCÍA LOMAS, G. ADRIANO (1922), Estudio del dialecto popular montañés. Fonética, etimologías y glosario de voces, San Sebastián.

- (1949), El lenguaje popular de las montañas de Santander. Fonética, recopilación de voces, refranes y modismos, $2^{\mathrm{a}}$ ed. muy ampliada, Santander: CSIC.

- (1966), El lenguaje popular de la Cantabria Montañesa. Fonética, recopilación de voces, juegos, industrias populares y modismos, $2^{\mathrm{a}}$ ed. revisada, Santander: Aldrés.

- (1977), Los pasiegos. Estudio crítico, etnográfico y pintoresco (Años 1011 a 1960), Santander: Ed. de librería Estilo. Es la segunda ed. de Los pasiegos. Estudio etnográfico e histórico, Santander, 1960.

GARCÍA MOUTON, PILAR (1987), «Dialectología y cultura popular. Estado de la cuestión», RDTP, XLII, págs. 49-74.

- (1990), «El estudio del léxico en los mapas lingüísticos», en Moreno Fernández, Francisco (recopilador) (1990), Estudios sobre variación linguiística, Alcalá de Henares: Universidad.

- (cd.) (1994), Geolinguiistica. Trabajos europeos, Madrid: C.S.I.C.

GIMENO MENÉNDEZ, FRANCISCO (1990), Dialectología y sociolingüística españolas, Alicante: Universidad.

GONZÁLEZ ECHEGARAY, JOAQUT́N Y DÍAZ GÓMEZ, ALBERTO (1988), Manual de Etnografía Cántabra, Santander: Ediciones de Librería Estudio.

HUIDOBRO, EDUARDO DE (1907), Palabras, giros y bellezas del lenguaje popular de la Montaña, Santander.

LAPESA, RAFAEL (1988), Historia de la lengua española, $9^{\mathrm{a}}$ ed. corregida y aumentada, Madrid: Gredos.

LAMANO BENEITE, JOSÉ DE (1989), Dialecto vulgar salmantino, Salamanca: Ediciones de la Diputación de Salamanca.

LÓPEZ VAQUÉ, ADOLFO (1988), Vocabulario de Cantabria (Apuntes para un vocabulario general), Santander.

MENÉNDEZ PIDAL, RAMÓN (1954), «Pasiegos y vaqueiros. Dos cuestiones de geogralía linguística», Archivum, IV, págs. 7-44.

- (1962), El dialecto leonés, prólogo, notas y apéndices de Carmen Bobes Naves, Oviedo: Instituto de Estudios Asturianos.

- (1973), El idioma español en sus primeros tiempos, $8^{a}$ ed., Madrid: EspasaCalpe. 
MIGUÉLEZ RODRÍGUEZ, EUGENIO, Diccionario de las hablas leonesas (León-Salamanca-Zamora), León, Ediciones Monte Casino, 1993.

MÚGICA, PEDRO DE (1892), Dialectos castellanos: montañés, vizcaino, aragonés, Berlín.

NAVARRO CARRASCO, ANA ISABEL (1993), «Geografía lingüística y diccionarios», ELUA, 9, págs. 73-96.

NEIRA, JESÚS Y PIÑEIRO, MARÍA ROSARIO (1989), Diccionario de los bables de Asturias, Oviedo: Instituto de Estudios Asturianos.

NUÑO ÁLVAREZ, MARÍA DEL PILAR (1996), «Cantabria» en Alvar, Manuel (dir.) (1996): págs. 183-196.

PENNY, RALF J. (1969), El habla pasiega: ensayo de dialectología montañesa, Londres: Tamesis Books.

- (1978), Estudio estructural del habla de Tudanca, Tübinger: Max Nimeyer Verlag.

— (1984), «Esbozo de un Atlas de Santander», LEA, VI/2, págs. 123-181.

R. A. E. (1992), Diccionario de la lengua española, 21 ${ }^{\mathrm{a}}$ ed., Madrid: Espasa-Calpe.

RENERO DÍAZ, VICENTE (1947), «Formas dialectales y toponímicas de Cantabria», Altamira, págs. 109-225.

RODRÍGUEZ CASTELLANO, LORENZO (1954), «Estado actual de la ' $h$ ' aspirada en la provincia de Santander», Archivum, IV, págs. 435-437.

- (1959) «Algunas precisiones sobre la metafonía en Santander y Asturias», Archivum, IX, págs. 236-247.

RUIZ NÚÑ̃Z, JOSÉ MANUEL (1998), El léxico agrícola según el Atlas de Cantabria, Alicante: Universidad.

SAIZ BARRIO, MIGUEL ÁNGEL (1991), Léxico cántabro, Santander: Ediciones Tantin.

SALVADOR, GREGORIO (1985), Semántica y lexicografia del español, Madrid: Paraninfo.

SALVADOR ROSA, AURORA (1985), «Las localizaciones geográlicas en el Diccionario de Autoridades», LEA, VII, págs. 103-139.

TOVAR, ANTONIO (1955), Cantabria prerromana o lo que la lingïística nos enseña de los antiguos cántabros, Madrid: Publicaciones de la Universidad Internacional «Menéndez Pelayo".

VERGARA MARTÍN, GABRIEL MARÍA (1921), «Materiales para la formación de un vocabulario de palabras usadas en Burgos y Santander», publicado como apéndice a su libro, Materiales para la formación de un vocabulario de palabras usadas en Segovia, Madrid.

ZAMORA VICENTE, A. (1972), Dialectología española, $2^{\mathrm{a}}$ ed. muy aumentada, Madrid: Gredos. 